\title{
TRANSFER OF UNCERTAINTIES THROUGH PHYSICAL-ACOUSTICAL-SONAR END-TO-END SYSTEMS: A CONCEPTUAL BASIS
}

\author{
A. R. ROBINSON ${ }^{1}$, P. ABBOT ${ }^{2}$, P.F.J LERMUSIAUX ${ }^{1}$, L. DILLMAN ${ }^{2}$ \\ ${ }^{1}$ Harvard University, Cambridge, MA 02138 USA \\ E-mail: robinson@pacific.deas.harvard.edu,pierrel@pacific.deas.harvard.edu \\ ${ }^{2}$ Ocean Acoustical Services and Instrumentation Systems (OASIS), Inc., 5 Militia Dr., Lexington, MA \\ 02421, USA \\ E-mail:abbot@oasislex.com,dillman@oasislex.com
}

\begin{abstract}
An interdisciplinary team of scientists is collaborating to enhance the understanding of the uncertainty in the ocean environment, including the sea bottom, and characterize its impact on tactical system performance. To accomplish these goals quantitatively an end-to-end system approach is necessary. The conceptual basis of this approach and the framework of the end-to-end system, including its components, is the subject of this presentation. Specifically, we present a generic approach to characterize variabilities and uncertainties arising from regional scales and processes, construct uncertainty models for a generic sonar system, and transfer uncertainties from the acoustic environment to the sonar and its signal processing. Illustrative examples are presented to highlight recent progress toward the development of the methodology and components of the system.
\end{abstract}

\section{Introduction}

The littoral environment can be highly variable on multiple scales in space and time, and sonar performance can be affected by these inherent variabilities. Uncertainties arise in estimates of oceanic and acoustic fields from imperfect measurements (data errors), imperfect models (model errors), and environmental variabilities not explicitly known. The focus of this paper is to present a conceptual basis to achieve the following: i) develop generic methods to efficiently characterize, parameterize, and prioritize system variabilities and uncertainties arising from regional scales and processes; ii) construct, calibrate, and evaluate uncertainty and variability models for the end-to-end system and it's components to address forward and backward transfer of uncertainties; and, iii) transfer uncertainties from the acoustic environment to the sonar and its signal processing in order to effectively characterize and understand sonar performance and predictions. In order to accomplish these objectives, an end-to-end system approach is necessary. 


\section{End-to-End System Approach}

An overview of the forward portion of our approach is shown by the bold arrows in Fig. 1, which connect the environment, acoustics and sonar to applications. The characterization and transfer of uncertainty begins with the environment, in particular from the spatial and temporal variability in the physical oceanography and spatial variability in the bottom.

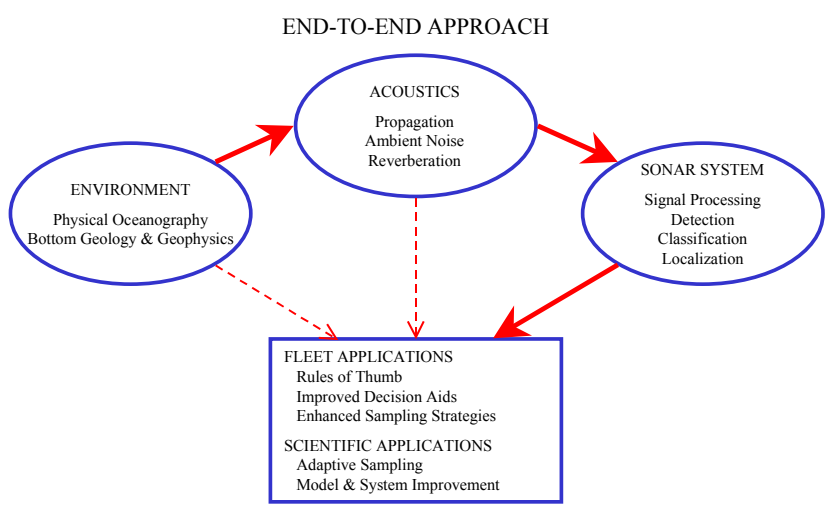
These effects impact the acoustics and result in uncertainties in acoustic predictions of propagation loss, reverberation and ambient noise. The uncertainties impact sonar performance, specifically, the ability of the system to detect a target. Ultimately, these methodologies can lead to improvements in scientific and fleet naval applications.

Figure 1. Overview of the end-to-end forward approach for capturing and transferring uncertainty.

The interactions among the different components of the system being more complex than Fig. 1 implies, it is only after multiple discussions on our respective disciplines and the corresponding information exchanges that a comprehensive and schematic picture of the end-to-end system crystallized. Fig. 2 schematizes the end-to-end system from the model point of view, where models are used to represent each of the coupled dynamics (boxes) and also the linkages to observation systems (circles). An effort was made to make the diagram exact but as simple as possible. The diagram illustrates the forward transfer of information, including uncertainties, in terms of observed, processed and model data (dots on arrows) and products and applications (diamond). The system concept encompasses the interactions and transfers of information with feedback from: i) observing systems, the information being physical-acoustical-bottom-noise-meteorological-sonar data, ii) coupled dynamical models, the information being physical-acoustical-bottom-noise-sonar state variables and parameters, and, iii) sonar equation models, the information being parameters in sonar equations. Note that the sonar system requirements configures the acoustic component and other system components for any particular problem and thereby sets the scales of uncertainty for the system.

Each of the coupled parameters and fields, i.e., the ocean environment, bottom characterization, noise field, acoustic state and sonar parameters, are classically described by mathematical equations; for example and respectively, by a primitive-equation model, Hamilton model, Wenz model, Helmoltz wave-equations and sonar equations (Sect. 3.2). These models are usually either static or dynamical, and deterministic or statistical. In addition to environmental noise, the sampling, processing and assimilation of data output 
from observational systems are also sources of uncertainty. First, sensor data are approximate. Second, platforms are of limited coverage in space and time and in variables measured. The linkages between data outputs from observational systems and the coupled model fields also involve substantial processing, thus uncertainties (Sect. 3.1). These linkages are represented by measurement models, which include error estimates. Data assimilation [14], combining data and model estimates to minimize uncertainties, is an integral part of our end-to-end system.

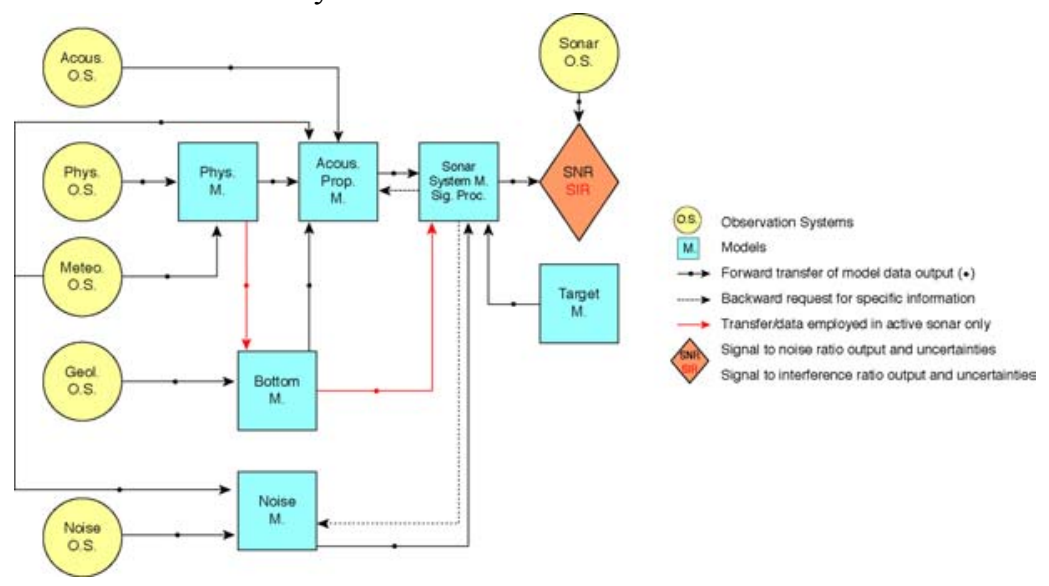

Figure 2. Schematic diagram of the end-to-end system (model point of view).
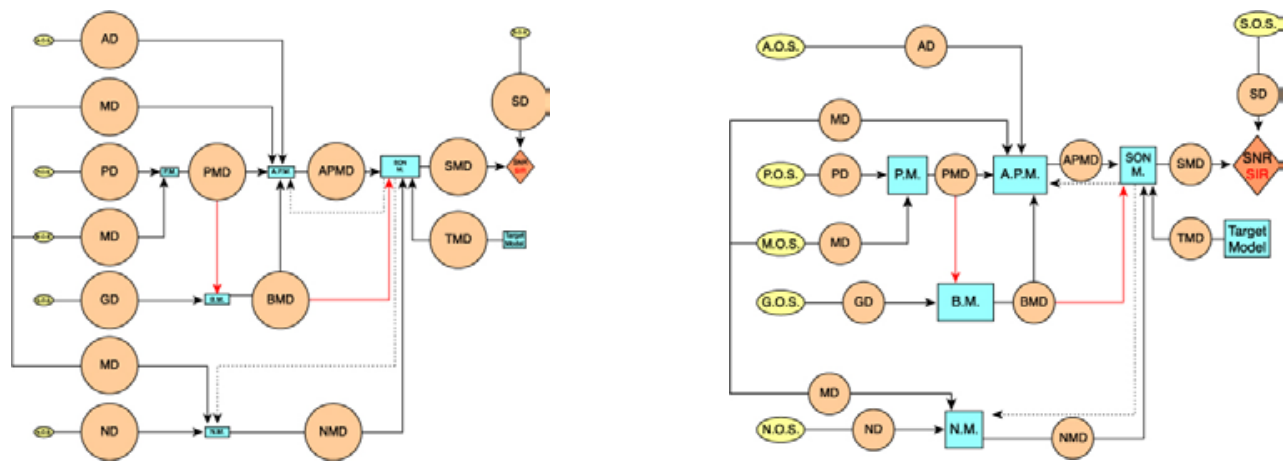

Figure 3. a) As Fig. 2 but from the data point of view; b) as Fig. 2, but with all (data + model) details.

In Fig. 2, each dot on an arrow corresponds to sets of estimated fields and of uncertainties. These information dots are enlarged on Fig. 3a; the detailed model and data diagram is on Fig. 3b. In these diagrams, uncertainties are defined by a representation of the likely errors in the estimated fields, usually in some probabilistic sense. Although uncertainties can be reduced, e.g. via data assimilation, there always remain some irreducible errors which need to be represented. There are several methodologies to represent errors 
and, for such complex interdisciplinary systems, efficient representations are an issue. An approach that we utilize (Sect. 4) is based on evolving an error subspace, of variable size, that spans and tracks the scales and processes where dominant errors occur. With this technique, Error Subspace Statistical Estimation (ESSE, [9,10,11]), the sub-optimal reduction of errors is itself optimal. Presently, the error subspace is initialized by decomposition on multiple scales and evolved in time by an ensemble of stochastic model iterations, where the stochastic terms represent model errors. The ensemble size is controlled by convergence criteria and a posteriori data residuals are employed for adaptive learning of the dominant errors [8]. This learning of errors from data misfits can be necessary because error estimates are themselves uncertain. In fact, a complete system likely represents each dot on an arrow (Fig. 2) as the sum of the: i) field, ii) its uncertainty and iii) the uncertainty on its uncertainty. Fuzzy information theories based on new uncertainty measures [7] and imprecise probability theories [16] are thus considered.

\section{End-to-End Components}

In this section, we discuss in more detail the end-to-end system components (Figs. 2, 3). The descriptions are limited to systems that may be employed in the current research and are not meant to be exhaustive. The present effort focuses on target detection performance.

\subsection{Observation Systems and Data}

Each observation system (OS on Figs. 2,3) corresponds to measurement models and uncertainties that describe the direct measurements, the sensors and procedures used to collect the data. In what follows, some examples are listed for illustrative purposes.

Acoustic Observation Systems Acoustic data are observed and processed to produce estimates such as transmission loss (TL), reverberation, scattering, attenuation etc. In our end-to-end system, we differentiate the direct observations from calculated acoustic parameters. Pertinent information, especially the uncertainty, regarding the collection of these data is contained by the AOS circle. The processed data and the method used to process the data, is included in the acoustic data products (data dot in Fig. 2).

Physical Observation Systems Physical data (temperature, salinity and velocity profiles) involve processing of raw measurements from XBTs, CTDs, ADCPs and current meters. Averaging, filtering, de-aliasing and calibration is necessary. Radar and satellite data (SSH, SST, etc.) also require processing, especially for use at high resolution in coastal regions.

Geological Observation Systems Geological data may be processed to produce data products used to characterize the ocean bottom properties such as bathymetry, bottom loss, bottom backscattering, sub bottom profiles, sediment thickness, sediment type, roughness, grain size, density and sound speed in the sediment, etc. Sensors and platforms include core grabbers, seismic surveys and source-receiver arrays.

Noise Observation Systems Although the noise measurements are usually collected jointly with acoustic measurements, it is differentiated here to allow individual assessments of this 
important parameter. Noise data may be processed to produce data products to characterize or calculate ambient noise as a function of depth and azimuth, shipping noise, shipping density, sea surface agitation and characteristics, etc.

Sonar Observation Systems Sonar observations are the observed SIR or SNR output of the sonar system and include the hardware as well as the signal processing characteristics of a given system. In our end-to-end system, the observed sonar SIR and SNR data are compared to modeled SIR and SIR that include the aggregate uncertainty.

\subsection{Coupled (Dynamical) Models}

The dynamical models and acoustical models are derived from the basic equations of the conservation of momentum, mass, heat, etc. for the fluid continuum (i.e. Navier-Stokes eqs.) appropriate approximations lead to the primitive equations for dynamics $[13\}$ and the Helmholtz equations for acoustics [14]. As discussed in [13,4] the ocean environment is a complex system that is inhomogeneous in three spatial dimensions and time. To completely describe this requires a conceptual model capable of handling the intricacies of the real ocean. For most applications further approximations are necessary. Numerical models discretize the approximate physical models for numerical results. Computations are subject to errors associated with the algorithms used and the limitations of the computer. A short overview of relevant coupled dynamical models (Figs. 2,3) is now provided.

Physical Models Physical oceanography encompasses a wide range of scales and considers processes from microstructure interactions to climate change. The focus here is on meso- and sub-mesoscale fronts and eddies, tides, internal tides, waves and solitons. Relevant dynamical models are then partial differential equations which include, in order of decreasing complexity: i) primitive-equation models, with or without a free ocean surface, ii) shallow-water models, iii) non-hydrostatic models, and iv) simplified time-independent balance equations such as the geostrophic equilibrium and thermal-wind balance. Most numerical ocean models are finite-difference, but some finite element models are also used. Only a few stochastic error models are employed, e.g. for representing uncertainties due to sub-mesoscale and internal tidal effects in mesoscale resolution models [11], but such stochastic research is growing.

Acoustic Propagation Models As reviewed in the literature (e.g. [4]), several propagation models may be used in this research. Most existing models are based on similar assumptions of boundary conditions and acoustic frequency, being all solutions or approximations to the Helmholz equation (linearized wave-equation). These models include Ray Models, Parabolic Equation Models, Normal Mode Models and Coupled Mode Models.

Bottom Models and Noise Models Bottom and sea-state surface models provide boundary conditions to acoustic models. Few studies have quantified the nature of the variability in bottom attribute (attenuation, reflectivity, velocity, density and proxies). Most geological bottom models, e.g. Hamilton model [5], are either a deterministic or a statistical representation of these attributes. For naval applications, several noise models are based on empirical and rule-of-thumb models, e.g. the Wenz [17] ambient noise spectra. However, 
this is an area of active research and complex models for the 3-D in space, frequencydependent, ambient noise (shipping, biologics and sea-surface noise) are being developed.

Sonar models We focus on target detection and the sonar model is represented by the sonar equations $[15,6]$ that describe signal excess (SIR or SNR) for an active or passive system. The sonar equations describe the interdependence of the environment, target, and sonar system and provide an estimate of the performance of the sonar system.

\section{Progress and Prospectus}

In this section, we illustrate the methodologies that we developed to: i) characterize and transfer environmental uncertainties to acoustical uncertainties [10]; and ii) characterize uncertainty as applied to transmission loss and sonar performance prediction.
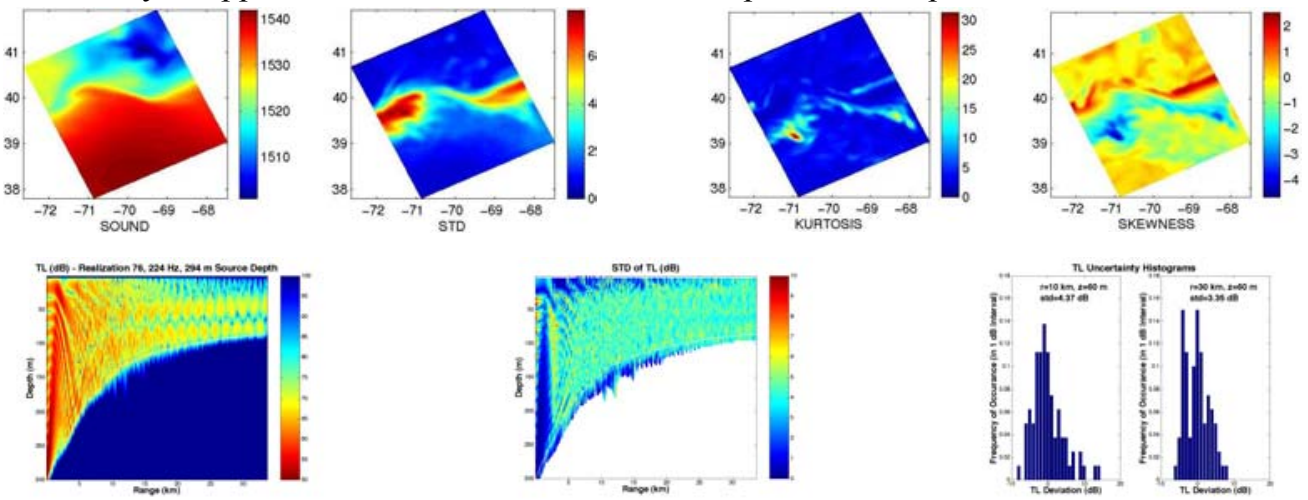

Figure 4. ESSE example of uncertainty transfer from the ocean physics to the acoustics.

Fig. 4 illustrates an ESSE simulation of the transfer of environmental uncertainty to acoustic prediction uncertainty in a shelfbreak environment. Based on observed oceanographic data during the 1996 Shelfbreak Primer Experiment, HOPS was initialized with perturbed fields that are in statistical accord with a realistic error subspace and then integrated to produce 80 realizations of a regional forecast of the sound speed field. The different realizations of the sound speed were then fed into a sound propagation model to produce realizations of the predicted TL for a low-frequency transmission from the slope, across the shelfbreak, onto the shelf. The ensemble mean, standard deviation, kurtosis and skewness of the sound speed at the surface level are shown (top). A realization of TL along the transmission path, its standard deviations and the histograms (PDF estimates) of TL uncertainties at two different locations (shelfbreak and shelf) are shown (bottom), revealing the complexity and inhomogeneity of the uncertainty statistics in this locale.

Next we discuss the use of uncertainty in the development of probabilistic performance predictions, using the metric predictive probability of detection (PPD). Figure 5a illustrates conceptually the PPD, details of which are given in [3]. The system-based environmental PDF is derived in our studies by a comparison of model predictions with system data. A 
histogram of the differences between the data and the acoustic model is fit with an appropriate distribution to yield the PDF. This PDF represents the uncertainty in the computational modeling process, typically small, and the inherent variability of the environment not contained in the model inputs, which typically is larger. The PPD is a prediction of the system performance versus range. Rather than use a single range value (e.g. "range-of-the-day" or "range-of-the-moment"), the PPD provides the system operator with a probabilistic representation of the system performance. The operator can thus use this information to operate the system more effectively, and can make more informed decisions on search, risk, and expenditure of assets.
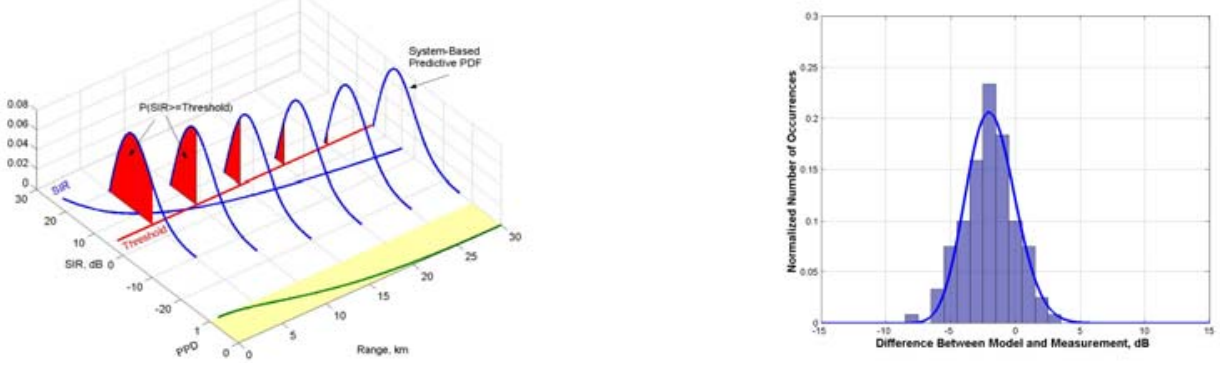

Figure 5a (left). Probabilistic system performance prediction and Predictive Probability of Detection (PPD) vs. range using System-Based Environmental PDF that incorporates environmental uncertainty.

Figure 5b (right). 1-Way TL Environmental PDF fit to histogram.

Fig. 5b illustrates an example of the 1-way TL environmental PDF based on operational experiments in shallow water [3]. This PDF quantifies the TL differences between predictions of the model and the truth of the data, caused by stochastic variability of the environment. Additional PDFs are presently being developed. These will include environmental (ambient noise, reverberation) and non-environmental (source level, target strength, system self-noise).

\section{Summary}

The end-to-end system encompasses the interactions and transfers of information with feedback from observing systems, coupled dynamical models that result in sonar performance predictions. The linkages and feedback among these different components are now being developed. The end-to-end framework is designed to support the individual components, environmental as well as non-environmental uncertainties (system related) so that an assessment of the dominant mechanism of uncertainty as it affects the SNR/SIR (Fig. 2) can be identified. The ability to assess the importance of the individual uncertainty components in the sonar performance prediction along within its aggregate uncertainty can be an invaluable tool in the development of tactical guidance. 


\section{Acknowledgments}

We thank the Office of Naval Research for support under grants N00014-00-1-0771 and contract N00014-00-D-0119, and W.G. Leslie for manuscript preparation. PFJL thanks Prof. C.-S. Chiu for his collaboration and M. Armstrong for figure preparation. This is a contribution of the UNITES team.

\section{References}

1. Abbot P., Celuzza S., Dyer I. Gomes B., Fulford J., Lynch J., Gawarkiewicz G., Volak D., Effects of Korean littoral environment on acoustic propagation. IEE Journal of Oceanic Engineering, 26, No. 2, 266-284 (2001).

2. Abbot P., Celuzza S., Dyer I. Gomes B., Fulford J. and Lynch J., Effects of East China Sea shallow water environment on acoustic propagation. IEE Journal of Oceanic Engineering, (submitted December 2001).

3. Abbot P. and Dyer I., Sonar Performance Predictions Based on Environmental Variability, Impact of Littoral Environmental Variability on Acoustic Predictions and Sonar Performance Conference Proceedings, NATO SACLANT Undersea Research Centre, to be published.

4. Chin-Bing S.A., Kink D.B. and Boyd J.D., The Effects of Ocean Environmental Variability on Underwater Acoustic Propagation Forecasting, Oceanography and Acoustics, Prediction and Propagation Models, American Institute of Physics Press, New York (1994).

5. Hamilton E., Geoacoustic Modeling of the Sea Floor, J. Acous. Soc. Am., Vol. 68, No.5 (1986).

6. Kinsler L., Frey A., Coppens A. and Sanders J., Fundamentals of Acoustics, 3rd edition. John Wiley \& Sons Inc., New York, 1982.

7. Klir G.J. and Wierman M.J., Uncertainty-based information. Elements of generalized information theory, 2nd edition, Studies in Fuzziness and Soft Computing, Physica-Verlag. (1999).

8. Lermusiaux P.F.J., Estimation and Study of Mesoscale Variability in the Strait of Sicily. Dynamics of Atmospheres and Oceans, Special Issue, 29, 255-303 (1999).

9. Lermusiaux P.F.J. and Robinson A.R., Data Assimilation via Error Subspace Statistical Estimation, Part I: Theory and Schemes. Monthly Weather Review, 127(8), 1385-1407 (1999).

10. Lermusiaux P.F.J., Anderson D.G. and Lozano C.J., On the Mapping of Multivariate Geophysical Fields: Error and Variability Subspace Estimates. Q.J.R. Meteorol Soc1387-1430 (2000).

11. Lermusiaux P.F.J., Chiu C.-S. and Robinson A.R., Modeling Uncertainties in the Prediction of the Acoustic Wavefield in a Shelfbreak Environment. Proceedings of the 5th International Conference on Theoretical and Computational Acoustics, May 21-25, 2001 (2002).

12. Robinson A.R., Physical Processes, Field Estimation and Interdisciplinary Ocean Modeling, Earth-Science Reviews 40, 3-54 (1996).

13. Robinson A.R. and Lee D., Ocean Variability, Acoustic Propagation and Coupled Models. Oceanography and Acoustics: Prediction and Propagation Models (A.R. Robinson and D. Lee, editors), American Inst. of Physics, New York, 1-6 (1994).

14. Robinson A.R., Lermusiaux P.F.J., and Sloan N.Q., Data Assimilation. In THE SEA: The Global Coastal Ocean, Volume 10: Processes and Methods (K.H. Brink and A.R. Robinson, eds.), John Wiley and Sons, NY, 541-594 (1998).

15. Urick Robert J., Principles of Underwater Sound, $3^{\text {rd }}$ edition. McGraw-Hill Book Company, New York (1983).

16. Walley P., Statistical Reasoning With Imprecise Probabilities, Monographs on Statistics and Applied Probability Series, No. 42 (1991).

17. Wenz G., Acoustic Ambient Noise in the Ocean: Spectra and Sources, J.Acous. Soc. Am. Vol. 34, No. 12 (1962). 